\title{
Prostaglandin E receptor subtype EP4 agonist serves better to protect cochlea than prostaglandin E1.
}

\section{AUTHOR(S):}

Hori, Ryusuke; Nakagawa, Takayuki; Yamamoto, Norio; Hamaguchi, Kiyomi; Ito, Juichi

\section{CITATION:}

Hori, Ryusuke ...[et al]. Prostaglandin E receptor subtype EP4 agonist serves better to protect cochlea than prostaglandin E1.. Auris nasus larynx 2013, 40(6): 539-542

\section{ISSUE DATE:}

2013-12

URL:

http://hdl.handle.net/2433/178677

\section{RIGHT:}

(c) 2013 Published by Elsevier Ireland Ltd.; この論文は出版社版でありま せん。引用の際には出版社版をご確認じ利用ください。; This is not the published version. Please cite only the published version. 
1 itle Page

2 Prostaglandin E Receptor Subtype EP4 Agonist Serves Better to Protect

3 Cochlea than Prostaglandin E1

4

5 RYUSUKE HORI ${ }^{1,2}$, TAKAYUKI NAKAGAWA ${ }^{1}$, NORIO YAMAMOTO ${ }^{1}$, KIYOMI

6 HAMAGUCHI ${ }^{1,2} \& \mathrm{JUICHI} \mathrm{ITO}^{1}$

7

$8{ }^{1}$ Department of Otolaryngology, Head and Neck Surgery, Graduate School of Medicine,

9 Kyoto University, Kyoto and ${ }^{2}$ Department of Otolaryngology, Tenri Hospital, Tenri, 10 Japan

11

12 Corresponding to Dr. Takayuki Nakagawa,

13 Department of Otolaryngology-Head and Neck Surgery, Graduate School of Medicine,

14 Kyoto University, Kawaharacho 54, Shogoin, Sakyo-ku, 606-8507 Kyoto, Japan.

15 E-mail: tnakagawa@ent.kuhp.kyoto-u.ac.jp

16 Tel: +81-75-751-3346 Fax: +81-75-751-7225

17

18 


\section{Introduction}

2 Prostaglandin E1 (PGE1) has long been clinically used as a vasodilator, and has been

3 proven to be effective for diverse circulatory disorders. Disorders associated with

4 cochlear blood flow have been considered some of the principle causes of sudden

5 sensorineural hearing loss (SSHL) [1], providing the rationale for the clinical use of

6 several vasodilators, including PGE1, for treatment of SSHL. Although PGE1 is a

7 therapeutic option for SSHL, its clinical benefit remains controversial [2-4]. PGE1

8 binds primarily to E-prostanoid receptors (EP) 1-4 [5], resulting in a variety of

9 biological effects, including vasodilation. In the central nervous system, some EP

10 signaling pathways mediate neurotoxic effects, but others, paradoxically, appear to

11 mediate protective effects [6]. Therefore, activation or inhibition of specific EPs might

12 have superior therapeutic potential than does PGE1 [6-8].

13 Previous studies have focused on the roles of EP4 in the cochlea and on cochlear

14 protection by pharmacological activation of EP4. EP4-deficient mice show slight

15 hearing loss and are susceptible to noise-induced hearing loss [9]. Local application of

16 an EP4 agonist has been shown to significantly attenuate noise-induced hearing loss in

17 mice [9] and guinea pigs [10]. These findings strongly suggest the superior potential of

18 EP4 agonists, compared with PGE1, for cochlear protection in clinic. However, 
1 comparative assessments of the efficacy of local application of EP4 agonists and PGE1,

2 which are crucial to precede clinical trials, have not been performed in cochlear

3 protection investigations. Therefore, this investigation aimed to examine whether an

4 EP4 agonist offered superior protective effects on cochleae, as compared with PGE1,

5 against noise trauma. For this investigation, a guinea pig model of noise-induced

6 hearing loss was used to compare the protective effects of ONO-AE1-437, an EP4

7 agonist, with those afforded byPGE1, following local application.

\section{$9 \quad$ Material and methods}

10 Experimental animals

11 Hartley guinea pigs, weighing 350-400g, were purchased from Japan SLC (Hamamatsu,

12 Japan). The Animal Research Committee of the Graduate School of Medicine, Kyoto

13 University, Japan, approved all of the experimental protocols. Animal care was

14 supervised by the Institute of Laboratory Animals of the Graduate School of Medicine,

15 Kyoto University. All experimental procedures involving animals were performed in

16 accordance with the National Institutes of Health's (USA) Guide for the Care and Use

17 of Laboratory Animals. 
2 The EP4 agonist, ONO-AE1-437, and PGE1, alprostadil (both from Ono

3 Pharmaceutical, Osaka, Japan) were applied to the round window of guinea pig

4 cochleae ( $\mathrm{n}=6$ for ONO-AE1-437, $\mathrm{n}=5$ for PGE1), as described previously [10]. In

5 our previous studies, ONO-AE1-329, a no-water soluble EP4 agonist was dissolved in

6 dimethyl sulfoxide followed by dilution in physiological saline, and locally

7 administered. As alprostadil is a water-soluble agent, a water-soluble EP4 agonist,

8 ONO-AE1-437 was chosen for the present study. Both agents were dissolved in

9 physiological saline to a final concentration of $1 \mathrm{mg} / \mathrm{mL}$. Under general anesthesia with

10 midazolam (10 mg/kg; intramuscular) and xylazine (10 mg/kg; intramuscular), the left

11 otic bulla of experimental animals was opened to expose the round window membrane.

12 A piece of gelatin, previously immersed in a solution of either the EP4 agonist or PGE1,

13 was placed on the round window membrane of each animal. For the animals in the

14 control group, a piece of gelatin immersed in physiological saline was applied $(\mathrm{n}=5)$.

$16 \quad$ Noise exposure and auditory brainstem response (ABR) recording

17 Immediately after drug application, animals were exposed to 4-kHz octave band noise at

18 120-dB sound pressure level (SPL) for 5 hours in a ventilated-sound exposure chamber 
1 fitted with speakers driven by a noise generator and a power amplifier. A 1/2-inch

2 condenser microphone and a fast Fourier transform analyzer (both from Sony, Tokyo,

3 Japan) were used to monitor and calibrate sound levels at multiple locations within the

4 chamber to ensure uniformity of the stimulus. The stimulus intensity varied by a

5 maximum of 3-dB SPL across the measured sites within the exposure chamber. ABRs

6 were recorded at frequencies of 4, 8, and $16 \mathrm{kHz}$ before noise exposure, and on Days 3,

7 7, 14, and 21 after exposure. The thresholds of the ABRs at each frequency were

8 determined, as described previously [10]. To test effects of local drug application on

9 hearing, ABR recording was performed in normal guinea pigs $(\mathrm{n}=4)$ following

10 placement of a piece of gelatin immersed in physiological saline on the round window

11 membrane.

\section{Histological assessment}

14 At the conclusion of the experiment (post-exposure Day 21), each cochlea was

15 subjected to histological analysis. Three regions of the cochlear sensory epithelia, at a

16 distances of $30-50 \%$ (second turn), $50-70 \%$ (mid-basal portion), and $70-90 \%$ (basal

17 portion) from the apex [11], were used for quantitative assessments of hair cell loss.

18 Immunohistochemical staining for myosin VIIa and F-actin labeling by phalloidin was 
1 conducted to label the inner hair cells (IHCs) and the outer hair cells (OHCs).

2 Anti-myosin VIIa rabbit polyclonal antibody (dilution, 1:500; Proteus BioSciences,

3 Ramona, CA, USA) was used as the primary antibody, and Alexa 568-conjugated goat

4 anti-rabbit immunoglobulin G (dilution, 1:500) was used as the secondary antibody.

5 After immunostaining for myosin VIIa, the specimens were stained with

6 fluorescein-phalloidin (1:400; Molecular Probes, Eugene, OR, USA) and examined by

7 confocal microscopy (TCS SP2; Leica Microsystems, Wetzlar, Germany). Nonspecific

8 labeling was tested by omitting the primary antibody from the staining procedures. The

9 numbers of IHCs and OHCs in 0.2-mm-long regions of the apical, middle, and basal

10 portions of the cochleae were independently counted by 3 investigators; the average of

11 the 3 counts was used in subsequent analyses.

13 Statistical analysis

14 The overall effects of applied drugs on ABR threshold shifts were examined using

15 two-way factorial analysis of variance with the post-hoc Fisher protected least

16 significant difference test (Fisher's PLSD). Differences in the numbers of IHCs and

17 OHCs in each region of cochleae were compared between experimental groups using

18 one-way factorial analysis of variance with Fisher's PLSD. $P$ values $<0.05$ were 
1 considered statistically significant. Values were expressed as the mean and the standard

2 deviation (SD).

3

4 Results

$5 \quad$ ABR threshold shifts

6 The time course of the ABR threshold shifts in noise-exposed animals at 4, 8, and 16

$7 \mathrm{kHz}$ are shown in Fig. 1. The overall effects of applied drugs were significant at 4, 8, or

$8 \quad 16 \mathrm{kHz}$ respectively $(\mathrm{p}<0.0001)$. The differences in the threshold shifts between the

$9 \quad$ EP4 agonist- and PGE1-treated cochleae were shown to be significant at 4, 8 or $16 \mathrm{kHz}$

10 ( $\mathrm{p}<0.0001)$, and those between the EP4 agonist- and saline-treated cochleae were

11 significant at 4,8 or $16 \mathrm{kHz}(\mathrm{p}<0.0001$ for 4 or $16 \mathrm{kHz}, \mathrm{p}=0.0004$ for $8 \mathrm{kHz})$. No

12 significant differences in the threshold shifts were found between the PGE1- and

13 saline-treated cochleae at each frequency $(\mathrm{p}=0.73,0.06,0.36$ for $4,8,16 \mathrm{kHz})$. No

14 significant elevation of ABR thresholds was found in normal guinea pigs after local

15 application of gelatin immersed in saline.

17 Histological assessment

18 Immunostaining for myosin VIIa and phalloidin staining for F-actin demonstrated 
1 severe degeneration of the OHCs in the second turn, mid-basal, and basal portions of

2 the PGE1-treated cochleae (Fig. 2A, C, E). By contrast, OHC degeneration was limited

3 in the EP4 agonist-treated cochleae (Fig. 2B, D, F). The IHCs were preserved in both

4 experimental groups (Fig. 2A-F). Quantitative assessments revealed significant

5 differences in the numbers of surviving OHCs among three groups in each portion (Fig.

$63 \mathrm{~A} ; \mathrm{p}=0.013,0.028,0.038$ for the second, mid-basal, basal portion). In the second

7 portion, significant differences in the numbers of surviving OHCs were found between

8 the PGE1- and saline-treated cochleae $(\mathrm{p}=0.039)$ and between the EP4 agonist- and

9 saline-treated cochleae $(\mathrm{p}=0.04)$. In the mid-basal and basal portion, the numbers of

10 surviving OHCs in the EP4-treated cochleae were significantly higher than those in the

11 PGE1- or saline-treated cochleae (Fig. 3A; $p=0.026$ or 0.012 for EP4 v.s. PGE1 or

12 saline in the mid-basal, $p=0.021$ or 0.028 for EP4 v.s. PGE1 or saline in the basal). No

13 significant differences were observed in the numbers of surviving IHCs among three

14 groups (Fig. 3B).

16 Discussion

17 The expression of four EP subtypes in the cochlea have been demonstrated [9, 10,

18 12-14], suggesting physiological or pathophysiological roles of EPs in the auditory 
1 function [8]. Previous studies have indicated the involvement of EP4 in the

2 physiopathology of cochleae and the therapeutic capability of EP4 agonists for

3 noise-induced hearing loss [9, 10], suggesting the potential of EP4 agonists as

4 therapeutic agents for acute sensorineural hearing loss. As PGE1 has also often been

5 used as a therapeutic option for SSHL, the ultimate goal of this experiment was to

6 provide preclinical evidence regarding an improved beneficial therapeutic option.

7 Before conducting a clinical trial to examine the efficacy of EP4 agonists for the

8 treatment of SSHL, demonstration of the differential efficacy of EP4 agonists and PGE1

9 in an animal model of acute sensorineural hearing loss was desirable. The present

10 results clearly demonstrated that the protective effects of an EP4 agonist on the cochleae

11 were superior to those of PGE1, both functionally and histologically, suggesting that

12 specific activation of EP4 can boost the therapeutic potential of PGE1 for SSHL.

13 Previously, we examined effects of the lipophilic EP4 agonist, ONO-AE1-329 on

14 noise-induced hearing loss in a guinea pig model and demonstrated significant

15 protection of cochleae in both pre- and post-traumatic treatment. The hearing gain

16 following post-traumatic treatment was significant, but not as obvious as seen in

17 pre-traumatic treatment. To clarify the difference in protective effects between a specific

18 EP4 agonist and PGE1, pre-traumatic treatment was employed in the present study. In 
1 the current study, a water soluble EP4 agonist, ONO-AE1-437 was investigated.

2 Considering its potential clinical application, the water-soluble nature may be

3 beneficial.

4 Both EP2 and EP4 induce intracellular production of cyclic adenosine monophosphate

5 (cAMP), while activation of EP3 results in decreased cAMP concentrations [6-8].

6 Therefore, EP2 agonists or EP3 antagonists might have similar effects on noise-induced

7 hearing loss, as did the EP4 agonist in this study, and this should be investigated in

8 future studies. In addition, it is also necessary to examine the effects of EP4 agonists in

9 other models of acute sensorineural hearing loss. Prior to clinical application of local

10 EP4 agonists for treatment of acute sensorineural hearing loss, determination of the

11 therapeutic treatment window will also need to be estimated.

12 In conclusion, the current study showed that local EP4 agonist treatment was superior to

13 local PGE1 treatment for the protection of auditory function and hair cells against

14 noise-induced damage in guinea pigs. Additional research will be required to translate

15 the findings of this study into recommendations for regular clinical application of EP4

16 agonists for the treatment of SSHL. 
1 This study was supported by a Grant-in-Aid for Scientific Research from the Ministry

2 of Education, Culture, Sports, Science and Technology of Japan, a Grant-in-Aid for

3 Research on Sensory and Communicative Disorders from the Japanese Ministry of

4 Health, Labour and Welfare and a collaborative research grant to Kyoto University from

5 Ono Pharmaceutical. We thank Ono Pharmaceutical Co., Ltd. for providing

6 ONO-AE1-437 and PGE1.

7

8 Conflict of interest: The authors report no conflicts of interest. The authors alone are

9 responsible for the content and writing of the paper. 


\section{References}

2

3 1. Nakashima T, Naganawa S, Sone M, Tominaga M, Hayashi H, Yamamoto H et al.

$4 \quad$ Disorders of cochlear blood flow. Brain Res Brain Res Rev 2003; 43: 17-28.

5 2. Ogawa K, Takei S, Inoue Y, Kanzaki J. Effect of prostaglandinE1 on idiopathic

6 sudden sensorineural hearing loss: a doubleblinded clinical study. Otol Neurotol 2002; 23:665-668.

8 3. Zhuo XL, Wang Y, Zhuo WL, Zhang XY. Is the application of prostaglandin E1

9 effective for the treatment of sudden hearing loss? An evidence-based meta-analysis.

$10 \quad$ J Int Med Res 2008; 36:467-70.

11 4. Agarwal L, Pothier DD. Vasodilators and vasoactive substances for idiopathic

12 sudden sensorineural hearing loss. Cochrane Database Syst Rev 2009; 4,

$13 \quad$ CD003422.

14 5. Sugimoto Y, Narumiya S. Prostaglandin E receptors. J Biol Chem. 2007; 282:11613-116137.

16 6. Andreasson K. Emerging roles of PGE2 receptors in models of neurological disease.

17 Prostaglandins Other Lipid Mediat 2010; 91, 104-112.

18 7. Legler DF, Bruckner M, Uetz-von Allmen E, Krause P. Prostaglandin E2 at new 
1 glance: novel insights in functional diversity offer therapeutic chances. Int $\mathrm{J}$

$2 \quad$ Biochem Cell Biol 2010; 42: 198-201.

3 8. Nakagawa T. Roles of prostaglandin E2 in the cochlea. Hear Res 2011; 276, 27-33

4 9. Hamaguchi K, Yamamoto N, Nakagawa T, Furuyashiki T, Narumiya S, Ito J. Role

5 of PGE-type receptor 4 in auditory function and noise-induced hearing loss in mice.

$6 \quad$ Neuropharmacology 2012; 62: 1841-1847.

7 10. Hori R, Nakagawa T, Sugimoto Y, Sakamoto T, Yamamoto N, Hamaguchi K, et al.

8 Prostaglandin E receptor subtype EP4 agonist protects auditory hair cells against

$9 \quad$ noise-induced trauma. Neuroscience 2009; 160: 813-819.

10 11. Viberg A, Canlon B. The guide to plotting a cochleogram. Hear Res 2004;197:1-10.

11 12. Stjernschantz J, Wentzel P, Rask-Andersen H. 2004. Localization of prostanoid receptors and cyclo-oxygenase enzymes in guinea pig and human cochlea. Hear Res 2004; 197: 65-73.

13. Hori R, Nakagawa T, Yamamoto N, Hamaguchi K, Ito J. Role of prostaglandin $\mathrm{E}$ receptor subtypes EP2 and EP4 in autocrine and paracrine functions of vascular endothelial growth factor in the inner ear. BMC Neurosci 2010;11:35.

17 14. Takumida M, Anniko M. Localization of prostanoid receptors in the mouse inner ear. Acta Otolaryngol 2011; 131:142-148. 


\section{Figure legends}

2 Fig. 1: Alterations in threshold shifts of the auditory brain-stem responses (ABRs) in

3 treated cochleae at frequencies of $4(\mathrm{~A}), 8(\mathrm{~B})$, and $16 \mathrm{kHz}(\mathrm{C})$. The overall effects of

4 applied drugs were significant at 4,8 or $16 \mathrm{kHz}(p<0.0001)$. The differences in ABR

5 threshold shifts at 4, 8, and $16 \mathrm{kHz}$ between the E-prostanoid receptor 4 (EP4) agonist-

6 and prostaglandin E1 (PGE1)- or saline-treated cochleae were significant (*). Bars

7 represent the SD.

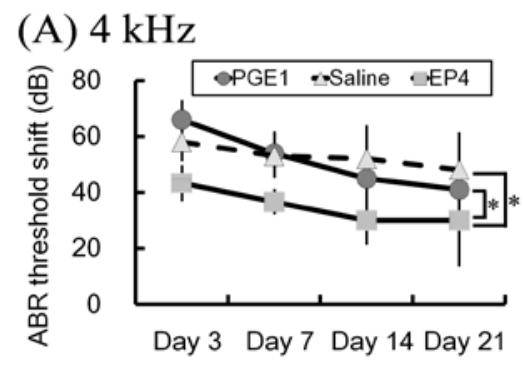

(B) $8 \mathrm{kHz}$

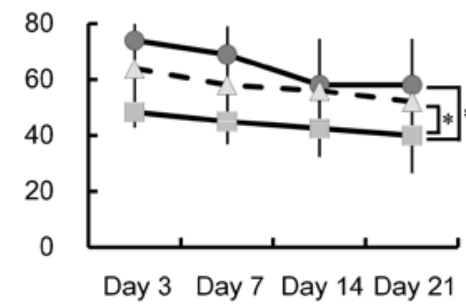

(C) $16 \mathrm{kHz}$

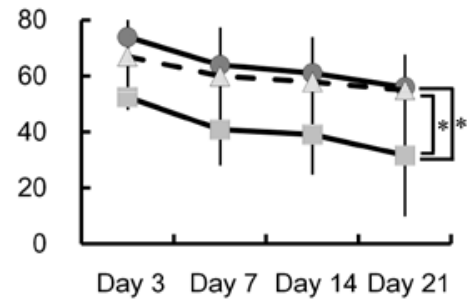

8

$9 \quad$ Fig. 2: Immunostaining for myosin VIIa (myo, red) and F-actin labeling with phalloidin

10 (pha, green) of cochlear sensory epithelia in the second turn, mid-basal, and basal

11 portions. Severe loss of outer hair cells (OHC) was observed in the prostaglandin

12 E1-treated cochlea (A, C, E). Degeneration of OHCs was limited in the specimen

13 treated with an E-prostanoid 4 receptor agonist (B, D, F). No significant difference in

14 inner hair cells (IHC) was observed between the 2 treatments. Bars represent $25 \mu \mathrm{m}$. 

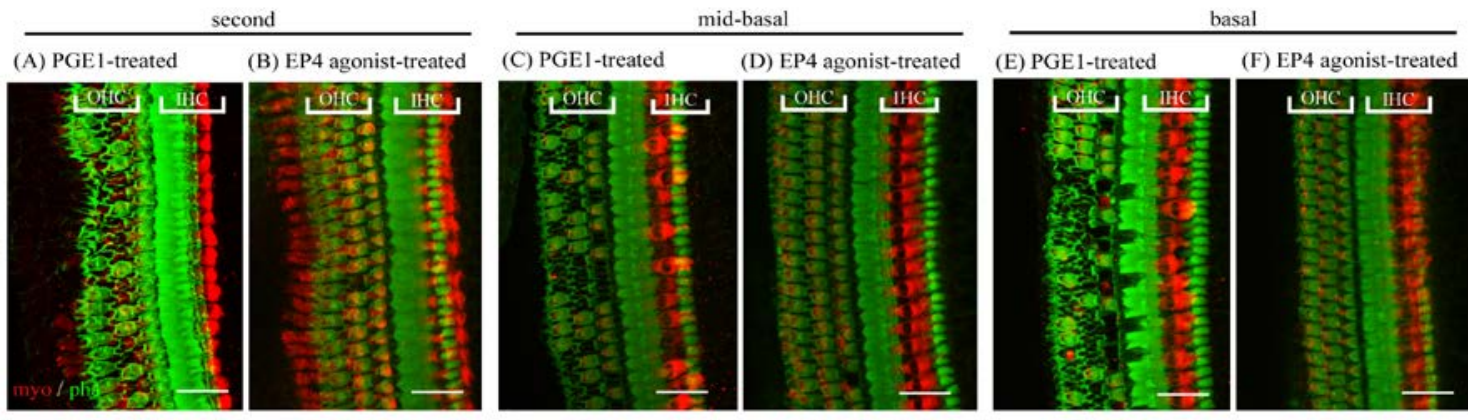

2 Fig. 3: Numbers of surviving outer (OHC, A) and inner hair cells (IHC, B) in the second,

3 mid-basal, and basal portions of treated cochleae. Asterisks indicate statistical

$4 \quad$ significance. Bars represent the SD.

\section{(A) $\mathrm{OHC}$}

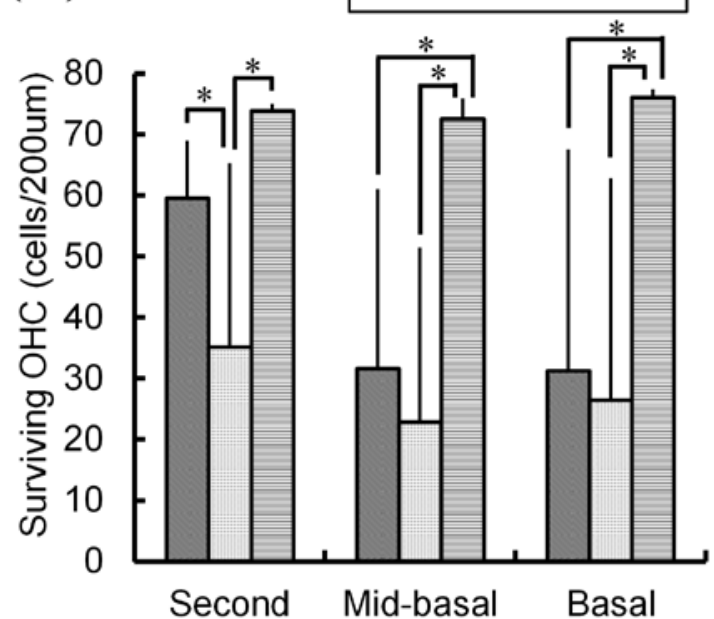

(B) IHC

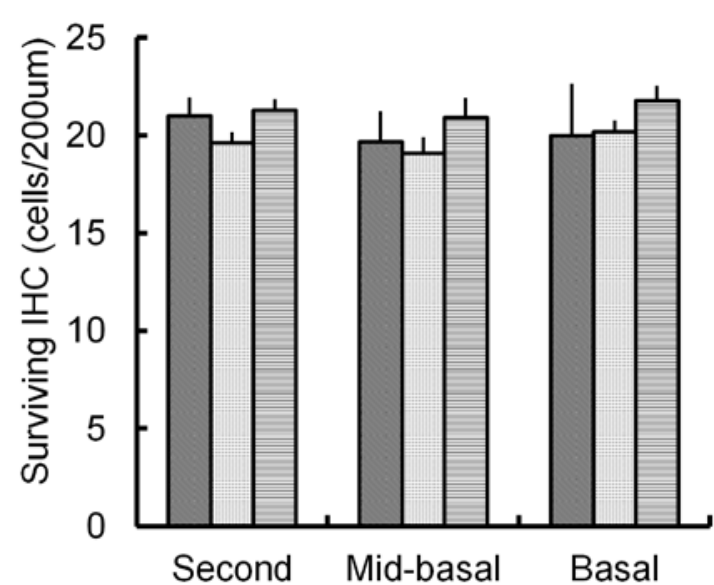

